\title{
Localization and identification of unknown target signal using oblique projection
}

\author{
Liping Huo ${ }^{1,3}$, Huijun $\mathrm{Hou}^{1,2}$ and Xingpeng $\mathrm{MaO}^{1,4,5^{*}}$ (D)
}

\begin{abstract}
The problem of source localization and waveform identification is the key of array signal processing. In this paper, an oblique projection-based localization and identification (OPLI) algorithm is proposed without known prior DOA or waveform information of the sources. The proposed OPLI is implemented iteratively. In each iteration, oblique projection is employed to separate the multiple incident signals into a series of single signal groups. After that, the procedure of waveform and DOA estimation for each single signal is implemented. Theoretical analysis and simulation result verify the performance and effectiveness of the proposed OPLI.
\end{abstract}

Keywords: Beamforming, Direction of arrival estimation, Oblique projection, Interference suppression

\section{Introduction}

Source localization and waveform identification are central problems in antenna array processing, including in particular radar, sonar, or wireless communication $[1,2]$. To this end, various sensor signal processing tools have been developed over the last several decades, ranging from direction-of-arrival (DOA) estimation algorithms to spatial beamforming algorithms [3-13]. It is noted that, in practical applications, the localization and identification of target signal could be difficult, when any a priori knowledge of signal-of-interest (SOI) is unavailable or the SOI is sheltered by adjacent interference signals. One of the general approaches to solve this problem is realized as follows: The DOA estimation is firstly implented for all the array receiving signals, and then, the waveform of $\mathrm{SOI}$ is obtained via beamforming techniques [14-19].

No matter in the process of DOA estimation or in beamforming, the influence of the interference signals on target localization and identification is generally not trivial, especially when the interference signals are spatially adjacent with the SOI in the presence of large power. If

*Correspondence: mxp@hit.edu.cn

'School of Electronics and Information Engineering, Harbin Institute of Technology, Harbin 150001, China

${ }^{4}$ Collaborative Innovation Centre of Information Sensing and Understanding, Harbin Institute of Technology, Harbin 150001, China

Full list of author information is available at the end of the article the interference signals are suppressed or attenuated in advance, the performance of localization and waveform identification for SOI would be better. Haimovich and Bar-Ness [20], Haimovich [21], and Honig and Goldstein [22] proposed eigenanalysis-based interference canceler. Similarly, Gu and Leshem [23], Huang et al. [24], Chan and Chen [25], Boyer [26], Xu et al. [27], Mak and Manikas [28], Shi and Lin [29], Behrens and Scharf [30], Mao et al. [31], and Mao et al. [32] employed beamforming techniques to suppress inference signals. Hassanien et al. [33], Vaccaro and Harrison [34], and Han and Zhang [35] designed matrix filters to cancel out-of-sector interference signals. It should be noted that these mentioned algorithms are based on accurate prior DOA of the SOI, and some of them may also require necessary DOA information of interference signals.

The estimation accuracy of DOA has major influence on the beamforming performances. Generally, the performance of a beamformer decreases severely when the DOA error increases [26, 31, 32]. Different approaches, including the linearly constrained minimum variance (LCMV), diagonal loading, convex optimization, and covariance matrix taper approaches, are developed $[16,36-46]$ to combat DOA errors. These algorithms can combat DOA uncertainties, but only suitable for small DOA errors. Lam and Singer [47], Bell et al. [48], 
and Han and Zhang [49] developed Bayesian beamforming, which is able to implement waveform identification when the DOA is uncertain or unknown, while it requires prior statistics that describes the level of DOA uncertainty.

Even if an accurate DOA of the SOI is given, the optimal performance of beamforming is hard to achieve since most of the beamformers rely on the sampled matrix inversion to replace the theoretical one, especially when in the presence of short data samples $[3,4,44,46]$. With limited number of snapshots, in this paper, we discuss the problem of localization and identification for unknown target signal, where there exist spatially adjacent interference signals and the DOAs of interference signals are also completely unknown. The well-known RELAX discuss the similar problem [50-52], where a simultaneous realization of DOA estimation and spatial beamforming for all receiving signals is achieved, but the performance of beamforming is not theoretically deduced.

With spatially adjacent interference signals, an oblique projection-based localization and identification (OPLI) algorithm is proposed for unknown target signals. Firstly, the OPLI employs oblique projection to separate the mixed array receiving signals into individual signal groups, where each signal group contains only one signal. Then, the OPLI sequentially estimates DOA and waveform of the signal in each group. Finally, the OPLI recursively reduces DOA and waveform errors via minimizing the optimal maximum likelihood cost function. Theoretical analysis is provided to show the beamforming performance of the proposed OPLI, and simulation results indicate that the OPLI is computationally effective for source localization and waveform identification; besides, it is superior to the counterpart conventional algorithms at moderate to high input signal-to-noise ratio (SNR) region in terms of output signal-to-interferencenoise ratio (SINR) and root mean square error (RMSE).

The paper is organized as follows. Section 2 presents the signal model for source localization and waveform identification. The proposed OPLI algorithm as well as its complexity analysis is developed in Section 3. Section 4 discusses the proposed OPLI, and Section 5 presents the simulation results. The conclusion is drawn in Section 7.

\section{Signal model and problem description}

\subsection{Signal model}

This subsection presents the signal model which is considered for source localization and waveform identification. Assume that $K$ far-field narrowband signals impinge upon an antenna array from distinct directions $\theta_{0}, \theta_{1}, \theta_{2}, \cdots, \theta_{K-1}$. The antenna array consists of $N$ sensors with arbitrary array geometry. The $N \times 1$ vector of received signals at time $t$ can be expressed as [3]

$$
\begin{aligned}
\mathbf{x}(t) & =\sum_{k=0}^{K-1} \mathbf{a}\left(\theta_{k}\right) s_{k}(t)+\mathbf{n}(t) \\
& =\mathbf{A S}(t)+\mathbf{n}(t)
\end{aligned}
$$

where

$$
\mathbf{A}=\left[\mathbf{a}\left(\theta_{0}\right), \mathbf{a}\left(\theta_{1}\right), \mathbf{a}\left(\theta_{2}\right), \cdots, \mathbf{a}\left(\theta_{K-1}\right)\right]
$$

and

$$
\mathbf{S}(t)=\left[s_{0}(t), s_{1}(t), s_{2}(t), \cdots, s_{K-1}(t)\right]^{\mathrm{T}}
$$

where $s_{k}(t)$ refers to the baseband signal waveform of the $k$ th incident signal, and $\mathbf{a}\left(\theta_{k}\right)$ denotes the corresponding steering vector. $\mathbf{n}(t)$ represents the additive noise component with covariance $\mathbf{R}_{n}=\mathbb{E}\left[\mathbf{n}(t) \mathbf{n}^{\mathrm{H}}(t)\right] . \mathbb{E}[\cdot],(\cdot)^{\mathrm{T}}$ and $(\cdot)^{\mathrm{H}}$ stand for statistical expectation, transposition and Hermitian transposition, respectively.

Both the received signals and the noise are assumed to be sampled from zero-mean and uncorrelated stationary random processes. Furthermore, the noise is temporally and spatially independent of the received signals, and $\mathbf{R}_{n}=\sigma^{2} \mathbf{I}$, where $\sigma^{2}$ is referred to as the noise power and $\mathbf{I}$ stands for the identity matrix. Thus, the covariance matrix of the received data is given by

$$
\mathbf{R}_{x}=\mathbb{E}\left[\mathbf{x}(t) \mathbf{x}^{\mathrm{H}}(t)\right]=\mathbf{A} \mathbf{R}_{S} \mathbf{A}^{\mathrm{H}}+\sigma^{2} \mathbf{I}
$$

where $\mathbf{R}_{S}=\mathbb{E}\left[\mathbf{S}(t) \mathbf{S}^{\mathrm{H}}(t)\right]$.

\subsection{Problem description}

This subsection formulates the localization and waveform identification problem. Without loss of generality, the signal $s_{0}(t)$ is assumed to be the SOI and the remaining $K-1$ received signals are treated as interference signals. For the SOI and the interference signals, neither the source locations (i.e., $\left.\theta_{1}, \theta_{2}, \cdots, \theta_{K-1}\right)$ nor the signal waveforms (i.e., $\left.s_{0}(t), s_{2}(t), \cdots, s_{K-1}(t)\right)$ are prior known.

The localization and waveform identification problem addressed in this paper is to obtain the DOA $\theta_{0}$ and waveform $s_{0}(t)$ from multiple snapshots $\left\{\mathbf{x}\left(t_{l}\right)\right\}_{l=1}^{L}$, where $L$ represents the number of snapshots.

In the next section, it will be shown that the waveform of the SOI can be identified as follows, i.e.,

$$
\hat{s}_{0}(t)=\mathbf{W}_{0}^{\mathrm{H}} \mathbf{x}_{0}(t)
$$

where $\mathbf{W}_{0}$ refers to the optimum weight, and

$$
\mathbf{x}_{0}(t)=\mathbf{a}\left(\theta_{0}\right) s_{0}(t)+\mathbf{n}(t) .
$$

And, according to (6), the location of the SOI is given by the conventional method of single target localization.

\section{Proposed OPLI algorithm}

Derivation and implementation of the proposed OPLI are presented in this section. The proposed OPLI attempts to employ oblique projection to separate the multiple incident signals into a series of single signal groups. As a 
result, the source localization and waveform identification are implemented on each separated single signal. Note that the procedure of oblique projection requires the DOA parameters for source separation. To this end, the OPLI is implemented iteratively and the method of maximum likelihood approximation [53] is employed to evaluate the convergence.

\subsection{Basic principle}

To illustrate the principle of localization and waveform identification of the SOI, the DOAs of all incident signals are temporarily assumed to be known in this subsection. According to (1) and (6), it is natural that the SOI component in the received data can be given by

$$
\begin{aligned}
\mathbf{x}_{0}(t) & =\mathbf{x}(t)-\sum_{k=1}^{K-1} \mathbf{a}\left(\theta_{k}\right) s_{k}(t) \\
& =\mathbf{x}(t)-\mathbf{B}_{0} \mathbf{S}_{\mathbf{B}_{0}}(t) .
\end{aligned}
$$

where

$$
\begin{aligned}
\mathbf{B}_{0} & =\left[\mathbf{a}\left(\theta_{1}\right), \mathbf{a}\left(\theta_{2}\right), \cdots, \mathbf{a}\left(\theta_{K-1}\right)\right] \\
& =\mathbf{A} \backslash \mathbf{a}\left(\theta_{0}\right)
\end{aligned}
$$

and

$$
\begin{aligned}
\mathbf{S}_{\mathbf{B}_{0}}(t) & =\left[s_{1}(t), s_{2}(t), \cdots, s_{K-1}(t)\right]^{\mathrm{T}} \\
& =\mathbf{S}(t) \backslash s_{0}(t)
\end{aligned}
$$

where the symbol $\backslash$ signifies set difference.

To separate the SOI from the multiple incident signals by (7), we employ oblique projection to obtain the interference signals, i.e.,

$$
\mathbf{S}_{\mathbf{B}_{0}}(t)=\mathbf{B}_{0}^{\dagger} \mathbf{E}_{\mathbf{B}_{0} \mid \mathbf{a}\left(\theta_{0}\right)} \mathbf{x}(t)
$$

where $\mathbf{E}_{\mathbf{B} \mid \mathbf{a}\left(\theta_{0}\right)}$ denotes the oblique projection operator whose range space is $\mathcal{R}\{\mathbf{B}\}$ and whose null space contains $\mathcal{R}\left\{\mathbf{a}\left(\theta_{0}\right)\right\}$, and

$$
\mathbf{E}_{\mathbf{B}_{0} \mid \mathbf{a}\left(\theta_{0}\right)}=\mathbf{B}_{0}\left[\mathbf{B}_{0}^{\mathrm{H}} \mathbf{P}_{\mathbf{a}\left(\theta_{0}\right)}^{\perp} \mathbf{B}_{0}\right]^{-1} \mathbf{B}_{0}^{\mathrm{H}} \mathbf{P}_{\mathbf{a}\left(\theta_{0}\right)}^{\perp}
$$

where

$$
\begin{gathered}
\mathbf{E}_{\mathbf{B}_{0} \mid \mathbf{a}\left(\theta_{0}\right)} \mathbf{B}_{0}=\mathbf{B}_{0} \\
\mathbf{E}_{\mathbf{B}_{0} \mid \mathbf{a}\left(\theta_{0}\right)} \mathbf{a}\left(\theta_{0}\right)=\mathbf{0}
\end{gathered}
$$

and

$$
\mathbf{P}_{\mathbf{a}\left(\theta_{0}\right)}^{\perp}=\mathbf{I}-\mathbf{a}\left(\theta_{0}\right) \mathbf{a}^{\dagger}\left(\theta_{0}\right)
$$

where $\mathcal{R}\{\cdot\},(\cdot)^{\perp}$, and $(\cdot)^{\dagger}$ denote range space, orthogonal complement, and Moore-Penrose pseudoinverse, respectively.

According to (7), only the SOI is contained in $\mathbf{x}_{0}\left(t_{l}\right)$, and the interferences signals are removed. Thus, the SOI can be estimated by solving the following minimization problem, i.e.,

$$
\left\{\hat{\theta}_{0}, \hat{s}_{0}(t)\right\}=\underset{\theta, s(t)}{\arg \min } \frac{1}{L} \sum_{l=1}^{L}\left\|\mathbf{x}_{0}\left(t_{l}\right)-\mathbf{a}(\theta) s\left(t_{l}\right)\right\|^{2}
$$

Solving (15), it is obtained that

$$
\hat{s}_{0}(t)=\mathbf{a}^{\dagger}\left(\theta_{0}\right) \mathbf{x}_{0}(t)
$$

and

$$
\begin{aligned}
\hat{\theta}_{0} & =\underset{\theta}{\arg \min } \frac{1}{L} \sum_{l=1}^{L}\left\|\mathbf{P}_{\mathbf{a}(\theta)}^{\perp} \mathbf{x}_{0}\left(t_{l}\right)\right\|^{2} \\
& =\underset{\theta}{\arg \min } \operatorname{tr}\left\{\mathbf{P}_{\mathbf{a}(\theta)}^{\perp} \mathbf{R}_{0}\right\} .
\end{aligned}
$$

where $\mathbf{P}_{\mathbf{a}(\theta)}^{\perp}=\mathbf{I}-\mathbf{a}(\theta) \mathbf{a}^{\dagger}(\theta)$, and $\mathbf{R}_{0}=\mathbb{E}\left[\mathbf{x}_{0}(t) \mathbf{x}_{0}^{\mathrm{H}}(t)\right]$.

Comparing (5) and (16), it follows that the optimum weight $\mathbf{W}_{0}$ to obtain the $\mathrm{SOI}$ is given by

$$
\mathbf{W}_{0}=\left[\mathbf{a}^{\dagger}\left(\theta_{0}\right)\right]^{\mathrm{H}}=\mathbf{a}\left(\theta_{0}\right)\left[\mathbf{a}^{\mathrm{H}}\left(\theta_{0}\right) \mathbf{a}\left(\theta_{0}\right)\right]^{-1}
$$

where $(\cdot)^{-1}$ denotes inverse.

Using (5), (7), (10), (17), and (18), both the location $\theta_{0}$ and the waveform $s_{0}(t)$ of the SOI can be computed. Besides, the localization and waveform identification processes for the SOI can also be extended, i.e., the localization and waveform identification process for the interference signals are feasible if each of the interference signals is regarded as the SOI.

\subsection{Practical considerations}

Recall that the localization and waveform identification process in Section 3.2 requires known DOAs of all the incident signals, which is infeasible in practical applications. In this paper, it is considered that the DOA of the $\mathrm{SOI}$ is prior unavailable, and the prior DOAs of the interference signals are also unknown. Toward the purpose, the presented localization and waveform identification process is implemented iteratively. The proposed OPLI firstly estimate the parameters of the SOI, so that a rough information of the SOI is available. And then, the parameters of each interference signals are updated one after another. Through iteration, not only the parameters of the SOI are estimated, but also the parameters of the interference signals are gradually achieving high precision.

At the $i$ th iteration, the estimated DOAs $\hat{\theta}_{0}^{(i)}, \hat{\theta}_{1}^{(i)}, \ldots$, $\hat{\theta}_{k-1}^{(i)}, \hat{\theta}_{k}^{(i-1)}, \hat{\theta}_{k+1}^{(i-1)}, \ldots, \hat{\theta}_{K-1}^{(i-1)}$ are utilized as initial values to compute $\hat{\theta}_{k}^{(i)}$ and $\hat{s}_{k}^{(i)}(t)$, where $i \geq 1$ and $k=$ $0,1,2, \cdots, K-1 . \hat{\theta}_{k}^{(i)}$ and $\hat{s}_{k}^{(i)}(t)$ denote the estimation of $\theta_{k}$ and $s_{k}(t)$ at the $i$ th iteration (similarly hereinafter), respectively. 
If the $k$ th incident signal is regarded as the SOI, using (7) (10) and (17), it follows that

$$
\hat{\theta}_{k}^{(i)}=\underset{\theta}{\arg \min } \operatorname{tr}\left\{\mathbf{P}_{\mathbf{a}(\theta)}^{\perp} \hat{\mathbf{R}}_{k}^{(i)}\right\}
$$

where

$$
\hat{\mathbf{R}}_{k}^{(i)}=\mathbb{E}\left[\hat{\mathbf{x}}_{k}^{(i)}(t)\left(\hat{\mathbf{x}}_{k}^{(i)}(t)\right)^{\mathrm{H}}\right]
$$

and

$$
\hat{\mathbf{x}}_{k}^{(i)}(t)=\mathbf{x}(t)-\hat{\mathbf{B}}_{k}^{(i)} \mathbf{S}_{\hat{\mathbf{B}}_{k}^{(i)}}(t)
$$

where

$$
\begin{gathered}
\mathbf{S}_{\hat{\mathbf{B}}_{k}^{(i)}}(t)=\left(\hat{\mathbf{B}}_{k}^{i}\right)^{\dagger} \mathbf{E}_{\hat{\mathbf{B}}_{k}^{(i)} \mid \mathbf{a}\left(\hat{\theta}_{k}^{(i-1)}\right)} \mathbf{x}(t) \\
\hat{\mathbf{B}}_{k}^{(i)}=\hat{\mathbf{A}}_{k}^{(i)} \backslash \mathbf{a}\left(\hat{\theta}_{k}^{(i-1)}\right)
\end{gathered}
$$

and

$$
\begin{aligned}
\hat{\mathbf{A}}_{k}^{(i)}= & {\left[\mathbf{a}\left(\hat{\theta}_{1}^{(i)}\right), \cdots, \mathbf{a}\left(\hat{\theta}_{k-1}^{(i)}\right),\right.} \\
& \left.\mathbf{a}\left(\hat{\theta}_{k}^{(i-1)}\right), \mathbf{a}\left(\hat{\theta}_{k+1}^{(i-1)}\right), \cdots, \mathbf{a}\left(\hat{\theta}_{K}^{(i-1)}\right)\right] .
\end{aligned}
$$

Besides, using (5) and (18), it follows that

$$
\hat{s}_{k}^{(i)}(t)=\left(\hat{\mathbf{w}}_{k}^{(i-1)}\right)^{\mathrm{H}} \hat{\mathbf{x}}_{k}^{(i)}(t)
$$

where

$$
\hat{\mathbf{w}}_{k}^{(i)}=\mathbf{a}\left(\hat{\theta}_{k}^{(i)}\right)\left[\left(\mathbf{a}\left(\hat{\theta}_{k}^{(i)}\right)\right)^{\mathrm{H}} \mathbf{a}\left(\hat{\theta}_{k}^{(i)}\right)\right]^{-1} .
$$

During the proposed iterative process, the relative change of the following cost function, which is derived from maximum likelihood approximation [53], between two consecutive iterations is utilized to indicate the convergence, i.e.,

$$
\mathcal{L}^{(i)}=\frac{1}{L} \sum_{l=1}^{L}\left\|\mathbf{x}\left(t_{l}\right)-\sum_{k=0}^{K-1} \mathbf{a}\left(\hat{\theta}_{k}^{(i)}\right) \hat{s}_{k}^{(i)}\left(t_{l}\right)\right\|^{2} .
$$

Referring to the well-known relaxed iterative approach in $[50,51]$, the localization and waveform identification procedure of the proposed OPLI is summarized in Table 1. It is shown that the proposed OPLI starts with the initialization $\mathbf{a}\left(\hat{\theta}_{0}^{(-1)}\right)=\mathbf{0}, \mathbf{a}\left(\hat{\theta}_{1}^{(-1)}\right)=\mathbf{0}, \cdots, \mathbf{a}\left(\hat{\theta}_{K-1}^{(-1)}\right)=\mathbf{0}$. At the $i$ th iteration, $\hat{\theta}_{j}^{(i)}$ is determined within the range scope $\left[\hat{\theta}_{j}^{(i-1)}-\epsilon_{j}, \hat{\theta}_{j}^{(i-1)}+\epsilon_{j}\right]$, where $K-1 \geq j \geq 0$, and $\epsilon_{j}$ is a user-selected parameter which indicates the range of the $j$ th signal in the spatial domain.

\subsection{Computational complexity}

In this subsection, the computational complexity of the proposed OPLI is analyzed.

Using $L$ number of snapshots, the computation of matrix $\hat{\mathbf{R}}_{k}^{(i)}$ in (20) can be given as $\sum_{l=1}^{L} \hat{\mathbf{x}}_{k}^{(i)}\left(t_{l}\right)$
Table 1 Iteration procedure of OPLI

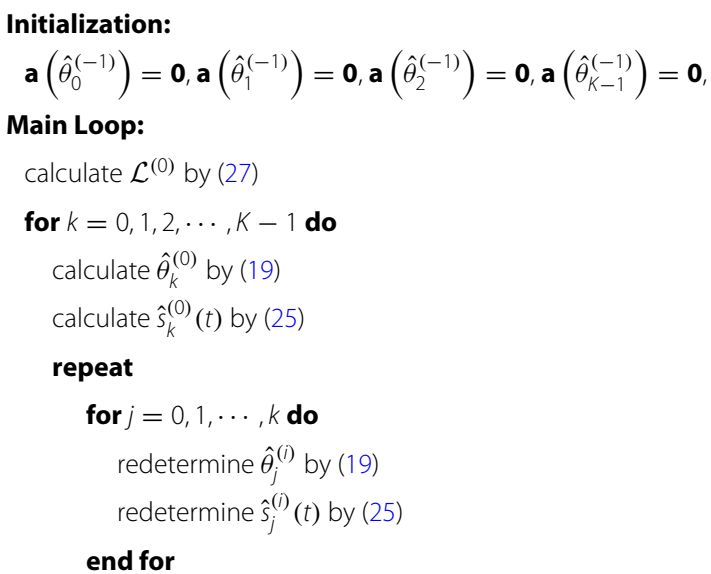

redetermine $\mathcal{L}^{(i)}$ by (27)

until $\left(\left|\mathcal{L}^{(i)}-\mathcal{L}^{(i-1)}\right|\right.$ is less than a specified tolerance)

$\mathbf{a}\left(\hat{\theta}_{0}^{(0)}\right) \leftarrow \mathbf{a}\left(\hat{\theta}_{0}^{(i)}\right), \mathbf{a}\left(\hat{\theta}_{1}^{(0)}\right) \leftarrow \mathbf{a}\left(\hat{\theta}_{1}^{(i)}\right), \cdots, \mathbf{a}\left(\hat{\theta}_{j}^{(0)}\right) \leftarrow \mathbf{a}\left(\hat{\theta}_{j}^{(i)}\right)$

end for

\section{Output}

$\hat{\theta}_{0} \leftarrow \hat{\theta}_{0}^{(i)}, \hat{\theta}_{1} \leftarrow \hat{\theta}_{1}^{(i)}, \ldots, \hat{\theta}_{K-1} \leftarrow \hat{\theta}_{K-1}^{(i)}$

$\left(\hat{\mathbf{x}}_{k}^{(i)}\left(t_{l}\right)\right)^{\mathrm{H}} / L$, which takes about $O\left(L N^{2}+N^{2}\right)$ flops. Herein, a flop is defined as a complex floating-point addition or multiplication operation. The number of flops roughly required to compute (21) is $O(N(K-1) L+N L)$ flops. The calculation of (22) requires approximately $O\left((K-1)^{3}+2 N(K-1)^{2}+N^{2}(K-1)+(K-1) N L\right)$ flops, where the calculation of $\mathbf{E}_{\hat{\mathbf{B}}_{k}^{(i)} \mid \mathbf{a}\left(\hat{\theta}_{k}^{(i-1)}\right)}$ additionally takes about $O\left(3 N^{2}(K-1)+2 N(K-1)^{2}+(K-1)^{3}+\right.$ $\left.2 N^{2}+N\right)$ flops. Thus, the computational complexity of $(20) \sim(22)$ is roughly $O\left(N^{2}(L+4 K-1)+\right.$ $\left.N\left(4(K-1)^{2}+(2 K-1) L+1\right)+2(K-1)^{3}\right)$ flops in total.

The computation of $\hat{\theta}_{k}^{(i)}$ in (19) requires roughly $O\left(\left(N^{3}+2 N^{2}+2 N\right) \tilde{N}_{\hat{\theta}_{k}^{(i)}}\right)$ flops, where $\tilde{N}_{\hat{\theta}_{k}^{(i)}}$ denotes the number of potential source locations in the region scope $\left[\hat{\theta}_{k}^{(i-1)}-\epsilon_{k}, \hat{\theta}_{k}^{(i-1)}+\epsilon_{k}\right]$. The computation of $\hat{s}_{k}^{(i)}(t)$ in (25) requires roughly $O(N L)$ flops, where the calculation of $\hat{\mathbf{W}}_{k}^{(i)}$ additionally takes about $O(2 N+1)$ flops. The computation of $\mathcal{L}^{(i)}$ in (27) requires roughly $O((K+2) N L)$ flops.

Therefore, according to Table 1, the computational complexity of the proposed OPLI is roughly $O((K+2) N L)+$ $O\left(\sum_{k=0}^{K-1}\left(\left(N^{3}+2 N^{2}+2 N\right) \tilde{N}_{\hat{\theta}_{k}^{(0)}}+N^{2}(L+4 K-1)+\right.\right.$ $N\left(4(K-1)^{2}+(2 K-1) L+1\right)+2(K-1)^{3}+N L+2 N+1+$ $\sum_{i=1}^{N_{r_{k}}}\left(\sum_{j=0}^{k}\left(\left(N^{3}+2 N^{2}+2 N\right) \tilde{N}_{\hat{\theta}_{j}^{(i)}}+N^{2}(L+4 K-1)+\right.\right.$ 
$N\left(4(K-1)^{2}+(2 K-1) L+1\right)+2(K-1)^{3}+N L+2 N$ $+1)+(K+2) N L))$ ) flops in total, where $N_{r_{k}}$ denotes the number of iterations employed in the $k$ th outer loop. Particularly, the total complexity is approximately $O\left(\sum_{k=0}^{K-1}\left(N^{3} \tilde{N}_{\hat{\theta}_{k}^{(0)}}+\sum_{i=1}^{N_{r_{k}}} \sum_{j=0}^{k} N^{3} \tilde{N}_{\hat{\theta}_{j}^{(i)}}\right)\right)$ flops, when $L \gg N>K, \tilde{N}_{\hat{\theta}_{k}^{(i)}} \gg L / N$, which occurs often in practical applications.

\section{Discussion}

The proposed OPLI involves the procedure of DOA estimation, hence it is applicable for localization of unknown target signal. Additionally, the OPLI also can obtain accurate locations of the interference signals, which is valuable in practical applications.

The proposed OPLI also involves the procedure of beamforming. Substituting (21), (22), (26) into (25), it follows that

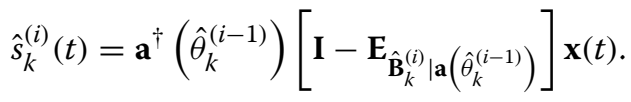

When OPLI achieves convergence, it follows from (28) that

$$
s_{k}(t)=\mathcal{W}_{\mathrm{opt}, k}^{\mathrm{H}} \mathbf{x}(t)
$$

where $\mathcal{W}_{\text {opt, } k}$ is referred to as the optimum beamformer employed by the proposed OPLI, $k=0,1,2, \cdots K-1$, and

$$
\mathcal{W}_{\text {opt }, k}=\left[\mathbf{a}^{\dagger}\left(\theta_{k}\right)\left[\mathbf{I}-\mathbf{E}_{\mathbf{B}_{k} \mid \mathbf{a}\left(\theta_{k}\right)}\right]\right]^{\mathrm{H}} .
$$

Let $k=0$, according to (2), (8), and (30), it is obtained that

$$
\mathcal{W}_{\text {opt }, 0}=\left[\mathbf{a}^{\dagger}\left(\theta_{0}\right)\left[\mathbf{I}-\mathbf{E}_{\mathbf{B}_{0} \mid \mathbf{a}\left(\theta_{0}\right)}\right]\right]^{\mathrm{H}}
$$

and

$$
\begin{gathered}
\mathcal{W}_{\mathrm{opt}, 0}^{\mathrm{H}} \mathbf{a}\left(\theta_{0}\right)=1 \\
\mathcal{W}_{\mathrm{opt}, 0}^{\mathrm{H}} \mathbf{B}_{0}=\mathbf{0} .
\end{gathered}
$$

Recall from [30] that

$$
\begin{aligned}
\mathbf{a}^{\dagger}\left(\theta_{0}\right) \mathbf{E}_{\mathbf{a}\left(\theta_{0}\right) \mid \mathbf{B}_{0}} & =\mathbf{a}^{\dagger}\left(\theta_{0}\right) \mathbf{P}_{\mathbf{a}\left(\theta_{0}\right)}\left[\mathbf{I}-\mathbf{E}_{\mathbf{B}_{0} \mid \mathbf{a}\left(\theta_{0}\right)}\right] \\
& =\mathbf{a}^{\dagger}\left(\theta_{0}\right)\left[\mathbf{I}-\mathbf{E}_{\mathbf{B}_{0} \mid \mathbf{a}\left(\theta_{0}\right)}\right]
\end{aligned}
$$

where

$$
\mathbf{E}_{\mathbf{a}\left(\theta_{0}\right) \mid \mathbf{B}_{0}}=\mathbf{a}\left(\theta_{0}\right)\left[\mathbf{a}^{\mathrm{H}}\left(\theta_{0}\right) \mathbf{P}_{\mathbf{B}_{0}}^{\perp} \mathbf{a}\left(\theta_{0}\right)\right]^{-1} \mathbf{a}^{\mathrm{H}}\left(\theta_{0}\right) \mathbf{P}_{\mathbf{B}_{0}}^{\perp} .
$$

Substituting (34) into (31), it is obtained that $\mathcal{W}_{\text {opt, } 0}=$ $\left[\mathbf{a}^{\dagger}\left(\theta_{0}\right) \mathbf{E}_{\mathbf{a}\left(\theta_{0}\right) \mid \mathbf{B}_{0}}\right]^{\mathrm{H}}$. So, the optimum beamformer of the proposed OPLI is equivalence to the well-known oblique projection beamformer which has excellent performance and has been well researched in $[26,31,32]$.
It follows from (31) to (33) that the proposed OPLI can effectively suppress interference signals and have distortionless response for the SOI. Regarding to the result of waveform identification for the SOI, the output SINR of OPLI is given by

$$
\operatorname{SINR}=\frac{\mathbb{E}\left[\left|\mathcal{W}_{\mathrm{opt}, 0}^{\mathrm{H}} \mathbf{a}\left(\theta_{0}\right) s_{0}(t)\right|^{2}\right]}{\mathbb{E}\left[\left|\mathcal{W}_{\mathrm{opt}, 0}^{\mathrm{H}}\left[\mathbf{x}(t)-\mathbf{a}\left(\theta_{0}\right) s_{0}(t)\right]\right|^{2}\right]} .
$$

Substituting (1), (31) (33) into (36), it follows that

$$
\begin{aligned}
\operatorname{SINR} & =\frac{\sigma_{0}^{2}}{\mathbb{E}\left[\left|\mathcal{W}_{\mathrm{opt}, 0}^{\mathrm{H}} \mathbf{n}(t)\right|^{2}\right]} \\
& =\frac{\sigma_{0}^{2}}{\mathcal{W}_{\mathrm{opt}, 0}^{\mathrm{H}} \mathbf{R}_{n} \mathcal{W}_{\mathrm{opt}, 0}}=\frac{\sigma_{0}^{2}}{\sigma^{2}\left\|\mathcal{W}_{\mathrm{opt}, 0}\right\|^{2}} \\
& =\frac{\sigma_{0}^{2}}{\sigma^{2}\left\|\mathbf{a}^{\dagger}\left(\theta_{0}\right)\left[\mathbf{I}-\mathbf{E}_{\mathbf{B}_{0} \mid \mathbf{a}\left(\theta_{0}\right)}\right]\right\|^{2}} \\
& =\frac{\sigma_{0}^{2}}{\sigma^{2} \| \mathbf{a}^{\dagger}\left(\theta_{0}\right) \mathbf{E}_{\mathbf{a}\left(\theta_{0}\right) \mid \mathbf{B}_{0} \|^{2}}} \\
& =\frac{\sigma_{0}^{2}}{\sigma^{2}} \mathbf{a}^{\mathrm{H}}\left(\theta_{0}\right) \mathbf{P}_{\mathbf{B}_{0}}^{\perp} \mathbf{a}\left(\theta_{0}\right)
\end{aligned}
$$

where $\sigma_{0}^{2}=\mathbb{E}\left[\left|s_{0}(t)\right|^{2}\right]$ refers to the power of the SOI.

It is known that the optimal SINR, which follows from the maximum SINR beamformer principle, is given by [46]

$$
\operatorname{SINR}_{\mathrm{opt}}=\sigma_{0}^{2} \mathbf{a}^{\mathrm{H}}\left(\theta_{0}\right) \mathbf{R}_{\mathrm{in}}^{-1} \mathbf{a}\left(\theta_{0}\right)
$$

where $\mathbf{R}_{\text {in }}^{-1}=\left(\mathbf{B}_{0} \mathbf{R}_{S_{\mathbf{B}_{0}}} \mathbf{B}_{0}^{\mathrm{H}}+\sigma^{2} \mathbf{I}\right)^{-1}$. Herein, $\mathbf{R}_{S_{\mathbf{B}_{0}}}=$ $\mathbb{E}\left[\mathbf{S}_{\mathbf{B}_{0}}(t) \mathbf{S}_{\mathbf{B}_{0}}^{\mathrm{H}}(t)\right]=\operatorname{diag}\left\{\left[\sigma_{1}^{2}, \sigma_{2}^{2}, \cdots, \sigma_{K-1}^{2}\right]\right\}$, where $\sigma_{k}^{2}$ denotes the power of the $k$ th signal. In particular, when the incident signals have equipower, i.e., $\sigma_{0}^{2}=\sigma_{1}^{2}=\cdots=$ $\sigma_{K-1}^{2}=\sigma_{\mathrm{i}}^{2}$, it is obtained that

$$
\mathbf{R}_{\mathrm{in}}^{-1}=\frac{1}{\sigma^{2}}\left(\frac{\sigma_{\mathrm{i}}^{2}}{\sigma^{2}} \mathbf{B}_{0} \mathbf{B}_{0}^{\mathrm{H}}+\mathbf{I}\right)^{-1}
$$

Further, if $\sigma_{\mathrm{i}}^{2} \gg \sigma^{2}$, then [30]

$$
\mathbf{R}_{\mathrm{in}}^{-1}=\frac{1}{\sigma^{2}} \lim _{\sigma_{\mathrm{i}}^{2} / \sigma^{2} \rightarrow \infty}\left(\frac{\sigma_{\mathrm{i}}^{2}}{\sigma^{2}} \mathbf{B}_{0} \mathbf{B}_{0}^{\mathrm{H}}+\mathbf{I}\right)^{-1}=\mathbf{P}_{\mathbf{B}_{0}}^{\perp} .
$$

Substituting (40) into (38), it is obtained that

$$
\begin{aligned}
\operatorname{SINR}_{\mathrm{opt}} & =\frac{\sigma_{0}^{2}}{\sigma^{2}} \lim _{\sigma_{\mathrm{i}}^{2} / \sigma^{2} \rightarrow \infty} \mathbf{a}^{\mathrm{H}}\left(\theta_{0}\right)\left(\frac{\sigma_{\mathrm{i}}^{2}}{\sigma^{2}} \mathbf{B}_{0} \mathbf{B}_{0}^{\mathrm{H}}+\mathbf{I}\right)^{-1} \mathbf{a}\left(\theta_{0}\right) \\
& =\frac{\sigma_{0}^{2}}{\sigma^{2}} \mathbf{a}^{\mathrm{H}}\left(\theta_{0}\right) \mathbf{P}_{\mathbf{B}_{0}}^{\perp} \mathbf{a}\left(\theta_{0}\right) .
\end{aligned}
$$


Comparing (41) with (37), it follows that the output SINR of the proposed OPLI can coincide well with the optimal SINR, when the power of the incident signals are equal and much higher than that of the noise.

\section{Simulation results}

Extensive simulation results are provided to verify the effectiveness of the proposed OPLI for source localization and waveform identification. The combinational algorithm, which firstly utilizes DOA estimator to obtain source locations and then utilizes beamformer for waveform identification, is compared with OPLI. Besides, the well-known RELAX [51] is also employed for performance comparison. Specially, for source localization, the stochastic Cramér-Rao bound (CRB) [54] is also used for performance evaluation.

Recall that the procedure of OPLI not only contains beamforming but also involves DOA estimation. The output SINR is employed for measuring the beamforming performance, and the RMSE is employed for evaluating the DOA estimation performance, where

$$
\text { RMSE }=\sqrt{\frac{1}{K M_{c}} \sum_{m=1}^{M_{c}} \sum_{k=1}^{K}\left|\hat{\theta}_{k, m}-\theta_{k}\right|^{2}}
$$

where $M_{c}$ denotes the number of Monte Carlo trials, $\hat{\theta}_{k, m}$ refers to the DOA estimation of $\theta_{k}$ in the $m$ th Monte Carlo trial (similarly hereafter). In the following simulations, $M_{c}=200$, and a half-wavelength spaced uniform linear array composed of $N=6$ sensors, is considered.

\subsection{Waveform identification}

This subsection evaluates the waveform identification performance of the proposed OPLI. The minimum variance distortionless response (MVDR) beamformer [36], the diagonally loaded sample matrix inversion (LSMI) beamformer [38], the general linear combination (GLC)based beamformer [39], and the optimal beamformer which is based on the maximum output SINR principle [46] are employed for performance comparison. The diagonal loading factor of the LSMI beamformer is set to be equal to the noise power.

Note that both OPLI and RELAX implement waveform identification without prior DOA information. Whereas the MVDR, LSMI, and GLC beamformers require prior DOA of the SOI to calculate beamforming weights. Unless otherwise stated, in the subsection, it is assumed that the prior DOA is considered without error.

Figure 1 presents the output SINR of the proposed OPLI, where the input SNR ranges from -20 to $30 \mathrm{~dB}$. $K=3$ signals are considered, and $L=200$ snapshots are employed. The SOI impinges on the array from $5.3^{\circ}$, and the interference signals are impinging from $-5.2^{\circ}$ and $45.5^{\circ}$. The simulation results show that the output SINR

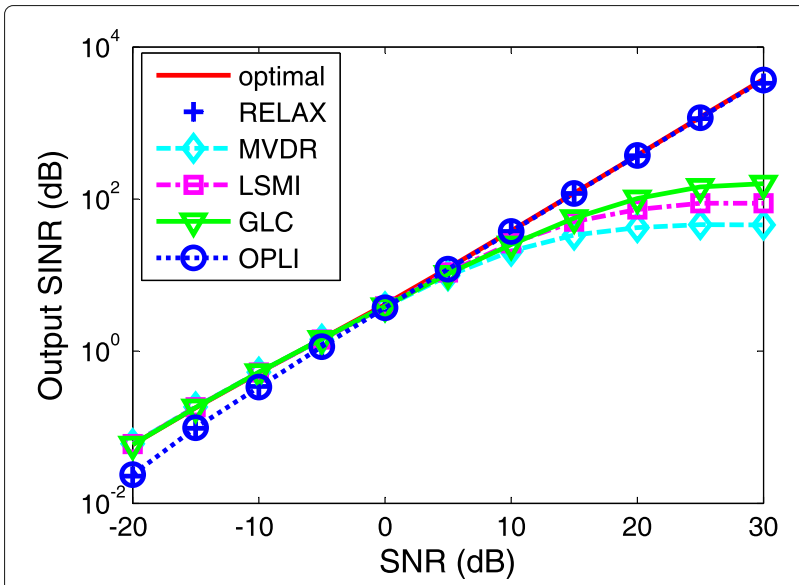

Fig. 1 Output SINR versus input SNR

of the proposed OPLI coincides well with the optimal output SINR when the input SNR within moderate to high region. Herein, RELAX shows a similar performance to the proposed OPLI. OPLI exhibits better performance than the counterpart MVDR, LSMI and GLC beamformers, and this is mainly due to the fact that OPLI employs oblique projection, which has excellent performance for suppressing interference signals, to identify the waveform of the SOI. The proposed OPLI tends to have a performance drop when the input SNR is lower than $0 \mathrm{~dB}$, this is because the employed oblique projection increases the noise variance (cf. $[26,30])$. The simulation result verifies the theoretical analysis given in Section 4.

Figure 2 presents the output SINR of the proposed OPLI, where the number of snapshots $L$ ranges from 2 to 1000 . The input SNR is fixed at $10 \mathrm{~dB}$. Other simulation conditions remain the same as previous experiment. It is seen that the output SINR gradually approximate to the optimal output SINR when the number of snapshots

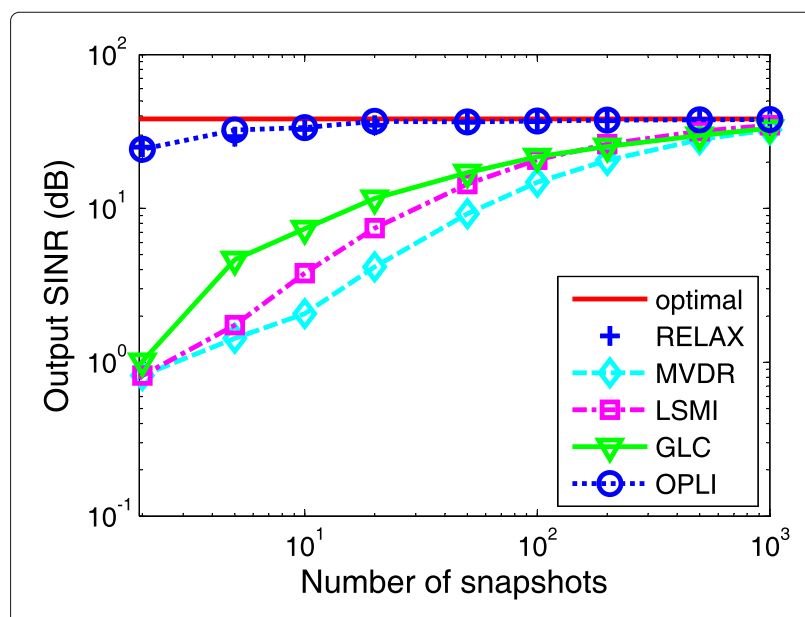

Fig. 2 Output SINR versus the number of snapshots 
increases. Compared to RELAX, OPLI exhibits the same performance. While comparing to the MVDR, LSMI, and GLC beamformers, the proposed OPLI has a better performance for waveform identification even if the number of snapshots is much limited.

Figure 3 presents the output SINR of the proposed OPLI, where the DOA error of the SOI ranges from $-5^{\circ}$ to $5^{\circ}$. In this simulation, $L=200$ snapshots are considered, and the input SNR is fixed at $10 \mathrm{~dB}$. Other simulation conditions remain the same as previous experiment, except that the prior DOA of the SOI is considered with different error for the MVDR, LSMI, and GLC beamformers. The prior DOA of the SOI is set to $5.3^{\circ}+\delta$, where $\delta$ is varied from $-5^{\circ}$ to $5^{\circ}$. The simulation results show that the MVDR, LSMI, and GLC beamformers are sensitive to the prior DOA uncertainties of the SOI. The larger the DOA error goes, the quicker the output SINR drops. Comparing to the MVDR, LSMI, and GLC beamformers, the proposed OPLI as well as RELAX remains the same output SINR. This is due to the fact that both RELAX and OPLI implement waveform identification without prior DOA information.

\subsection{Source localization}

This subsection evaluates the source localization performance of the proposed OPLI. The well-known RELAX [51], multiple signal classification (MUSIC) [55], and perturbed SBL (PSBL) [56] are included for performance comparison.

Figure 4 presents the RMSE of the DOA estimates for the proposed OPLI, where the input SNR ranges from -20 to $20 \mathrm{~dB} . K=3$ signals impinging from $-5.2^{\circ}, 5.3^{\circ}$, and $45.5^{\circ}$ are considered, and $L=200$ snapshots are employed. It is seen that the RMSE of the proposed OPLI coincides well with the CRB within a moderate to high SNR region. Compared to PSBL, the proposed OPLI has

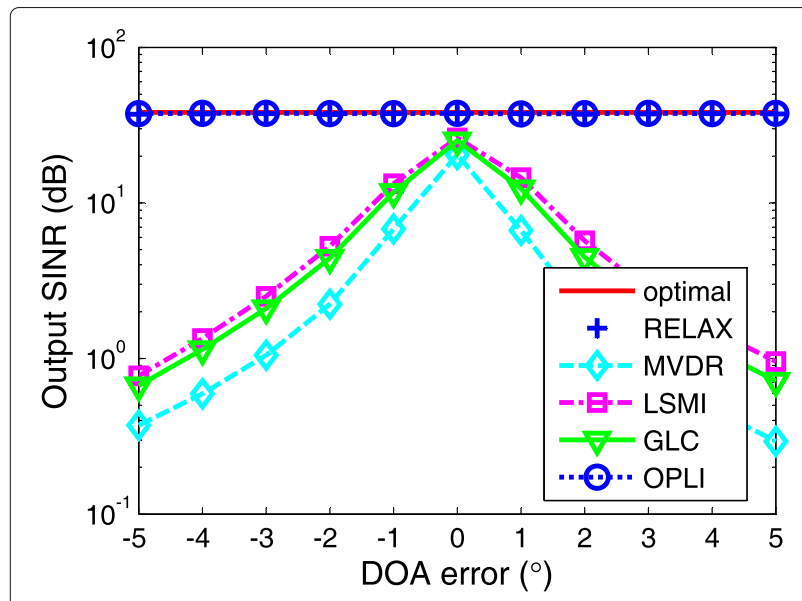

Fig. 3 Output SINR versus DOA uncertainty of the SOI

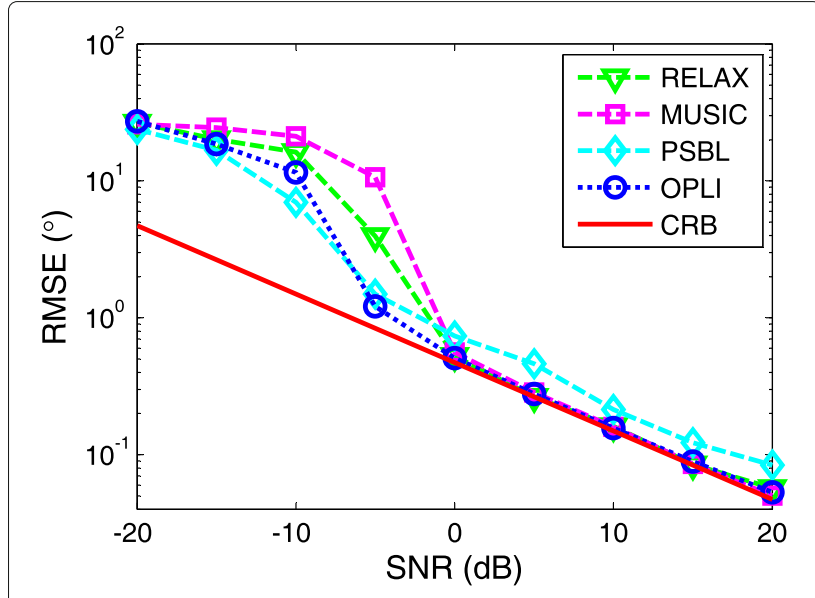

Fig. 4 RMSE versus input SNR

a much better performance when the input SNR is high Compared to MUSIC and RELAX, the proposed PSBL exhibits a better performance when the input SNR is low.

Figure 5 verifies the influence of the number of snapshots on DOA estimation, where the number of snapshots $L$ is varied from 1 to $400 . K=3$ signals impinging from $-5.2^{\circ}, 5.3^{\circ}$, and $45.5^{\circ}$ are considered, and the input SNR is fixed at $15 \mathrm{~dB}$. It can be seen that the proposed OPLI as well as RELAX is computationally efficient for DOA estimation even if the number of snapshots is small, whereas the conventional MUSIC fails in this case. Compared to PSBL, the DOA estimation performance of the proposed OPLI is more attractive. When the number of snapshots increases, the RMSE of the proposed OPLI decreases. The RMSE of OPLI, MUSIC, and RELAX can coincide well with the CRB when a large number of snapshots is given.

Figure 6 examines the influence of angular separation on DOA estimation, where $K=2$ signals impinging from $5.3^{\circ}-\Delta$ and $5.3^{\circ}$ are considered. $\Delta$ ranges from

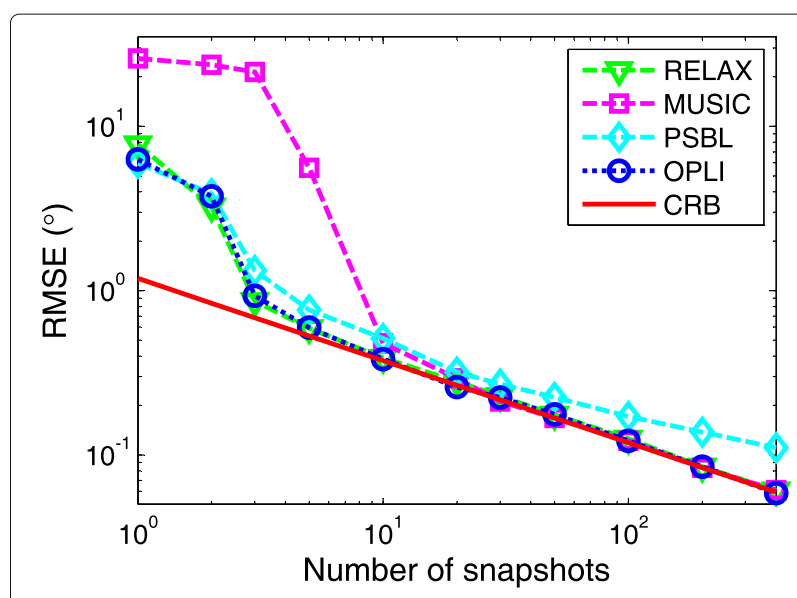

Fig. 5 RMSE versus the number of snapshots 


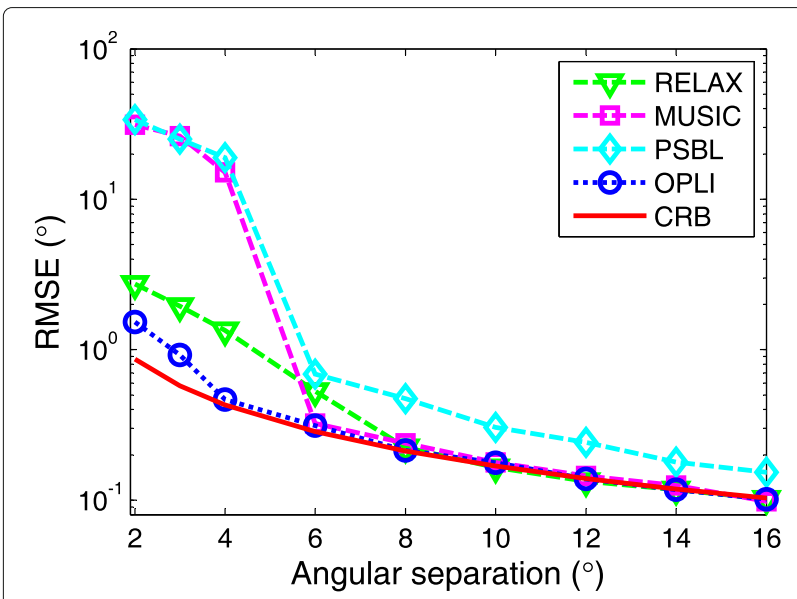

Fig. 6 RMSE versus angular separation

$2^{\circ}$ to $16^{\circ}$, the input $\mathrm{SNR}$ is fixed at $10 \mathrm{~dB}$, and $L=200$ snapshots are employed. The simulation results indicate that the RMSE of OPLI coincides well with the CRB when the angular separation is large. Furthermore, the proposed OPLI exhibits a better DOA estimation performance than RELAX, MUSIC and PSBL when the angular separation is small. OPLI exhibits a better performance for source localization of spatially adjacent sources than the counterpart algorithms.

\subsection{Comprehensive performance evaluation}

This subsection compares the comprehensive localization and waveform identification performances of the proposed OPLI with that of the combinational algorithms. Here, the combinational algorithms employ MUSIC and PSBL to estimate DOA of the SOI, and utilize MVDR, LSMI, and GLC to estimate the waveform of the SOI. For comprehensive performance evaluation, the average root mean square error (AveRMSE) is utilized, and it is defined as

$$
\begin{aligned}
\text { AveRMSE }= & \frac{1}{2}\left(\sqrt{\frac{1}{M_{c}} \sum_{m=1}^{M_{c}} \sum_{l=1}^{L}\left|\hat{s}_{0, m}\left(t_{l}\right)-s_{0}\left(t_{l}\right)\right|^{2} / \sum_{l=1}^{L}\left|s_{0}\left(t_{l}\right)\right|^{2}}\right. \\
& \left.+\sqrt{\frac{1}{M_{c}} \sum_{m=1}^{M_{c}}\left|\hat{\theta}_{0, m}-\theta_{0}\right|^{2}}\right) .
\end{aligned}
$$

Besides, the computational complexity of the proposed OPLI, which is evaluated by the running time of algorithm, is also compared with those of the combinational algorithms.

Figure 7 illustrates the AveRMSE of the proposed OPLI, where the input SNR ranges from -20 to $20 \mathrm{~dB}$. $K=3$ signals are considered, and $L=200$ snapshots are employed. The SOI is incident from $5.3^{\circ}$, and the interference signals is incident from $-5.2^{\circ}$ and $45.5^{\circ}$. From the

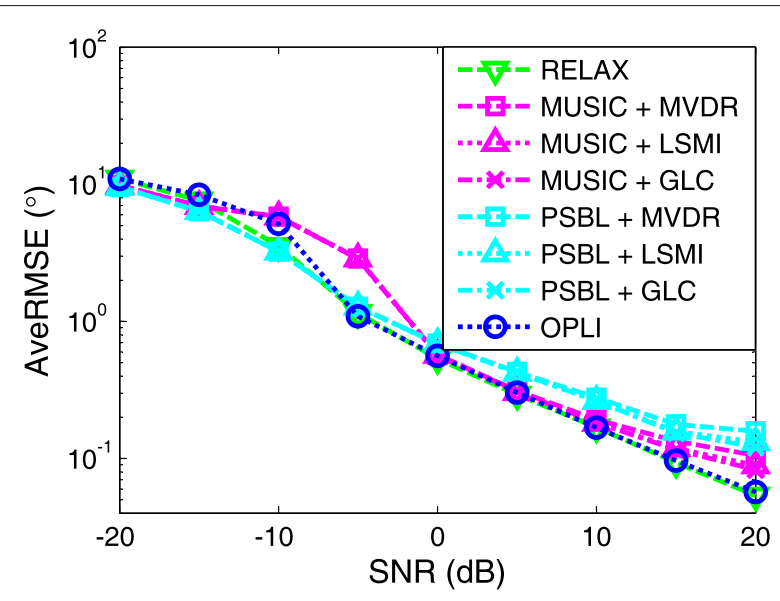

Fig. 7 AveRMSE versus input SNR

simulation results, it is seen that the proposed OPLI and the well-known RELAX exhibit better performance than the counterpart algorithms, when the input SNR within moderate to high region. With a low input SNR, the performance of the proposed OPLI is not attractive, and this performance verifies the result of Fig. 1.

Figure 8 illustrates the AveRMSE of the proposed OPLI, where the number of snapshots ranges from 1 to $400 . K=3$ signals are considered, where the $\mathrm{SOI}$ is incident from $5.3^{\circ}$ and the interference signals is incident from $-5.2^{\circ}$ and $45.5^{\circ}$. The input SNR is fixed at $15 \mathrm{~dB}$. It follows from the simulation results that the proposed OPLI and the well-known RELAX exhibit a better comprehensive performance than the other counterpart combinational algorithms, when the number of snasphots is much limited. With a few number of snasphots, the performance of the proposed OPLI is much attractive.

Figure 9 illustrates the AveRMSE of the proposed OPLI, where different angular separation is considered. $K=2$

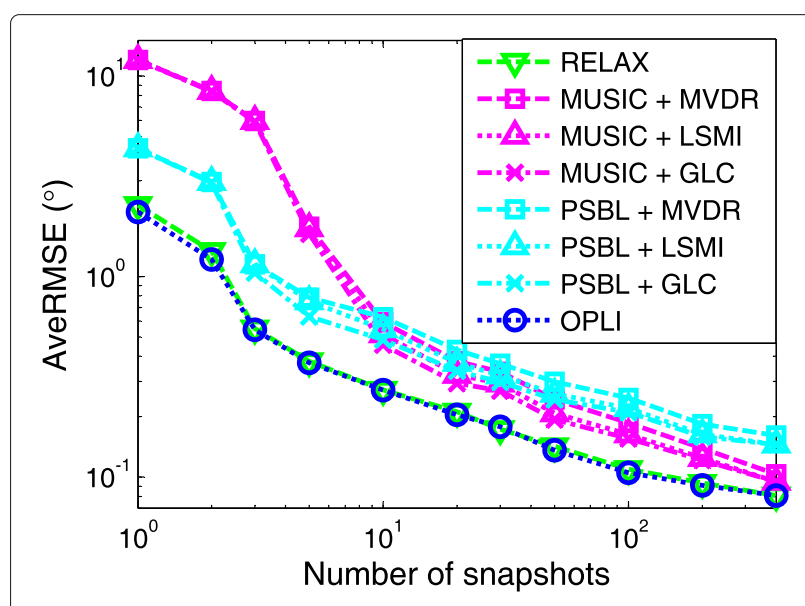

Fig. 8 AveRMSE versus the number of snapshots 


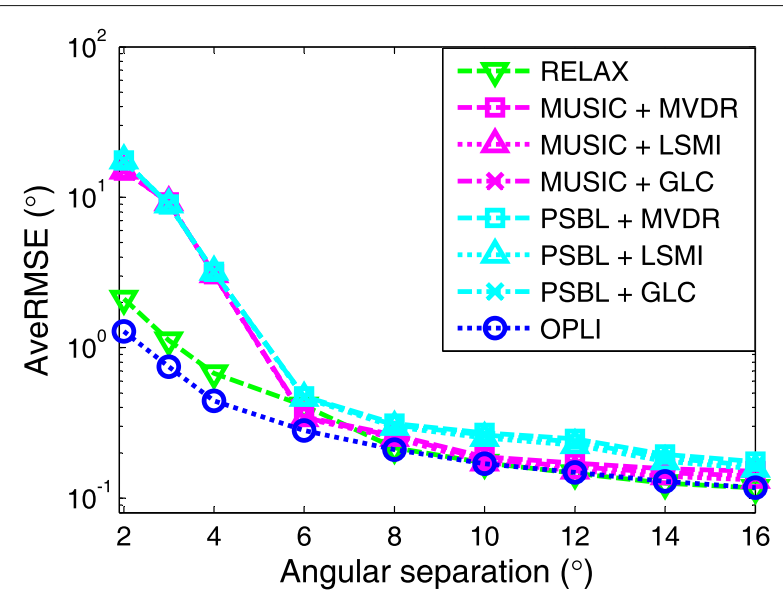

Fig. 9 AveRMSE versus angular separation

signals are considered, the input SNR is fixed at $10 \mathrm{~dB}$, and $L=200$ snapshots are employed. The SOI impinges from $5.3^{\circ}$, and the interference signals impinge from $5.3^{\circ}-\Delta$, where $\Delta$ ranges from $2^{\circ}$ to $16^{\circ}$. It can be seen that the proposed OPLI is superior to the well-known RELAX when the angular separation is small. With a small angular separation, comparing to the counterpart combinational algorithms, the proposed OPLI also shows a better performance, and this is mainly because the DOA estimation accuracy of the proposed OPLI is much higher than those of the counterpart algorithms (see also Fig. 5).

Figure 10 shows the running time of the proposed OPLI at each input SNR. Figures 11 and 12 illustrate the running time of the proposed OPLI when different number of snapshots and different angular separations are correspondingly considered. Parameters settings of Figs. 10, 11, and 12 remain the same as Figs. 7, 8, and 9, respectively. All the simulation experiments are performed using

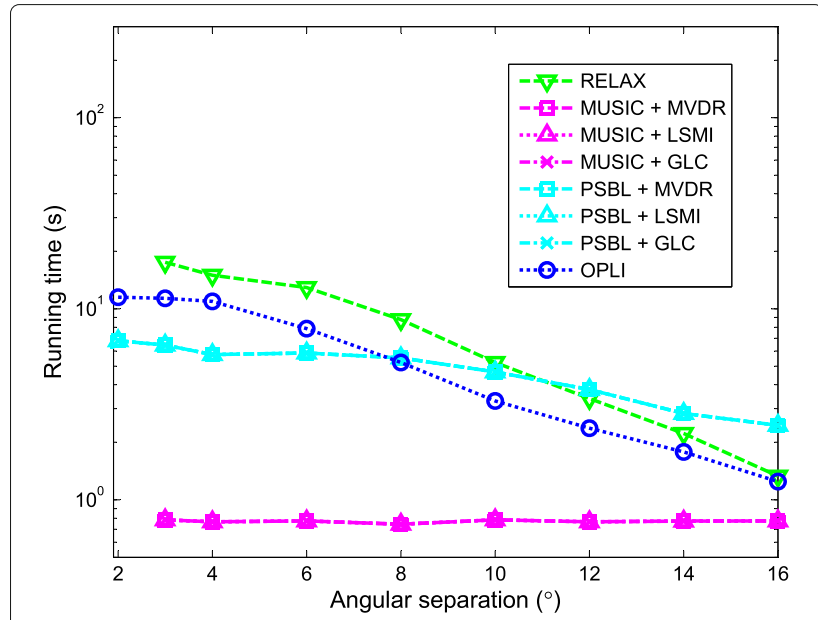

Fig. 11 Running time versus the number of snapshots

MATLAB 2013b running on a computer with a $2.3 \mathrm{GHz}$ Intel Quad-Core processor and 8GB RAM, under Windows 8.1. It can be seen that the computational complexity of the proposed OPLI is lower than that of the RELAX, but is higher than that of the MUSIC-based combinational algorithms. This is mainly because the localization and waveform identification processes of the proposed OPLI is implemented iteratively, whereas the MUSICbased combinational algorithms are not. Compared with the PSBL-based combinational algorithms, the proposed OPLI illustrates a relatively stable running time when the input SNR and the number of snapshots within moderate to high SNR region. However, when the SNR, the number of snapshots and the angular separation increase, the running time of the PSBL-based combinational algorithms fluctuates. This is due to the fact that the iteration number of PSBL is not stable and keeps varying with these parameters (cf. [56]).

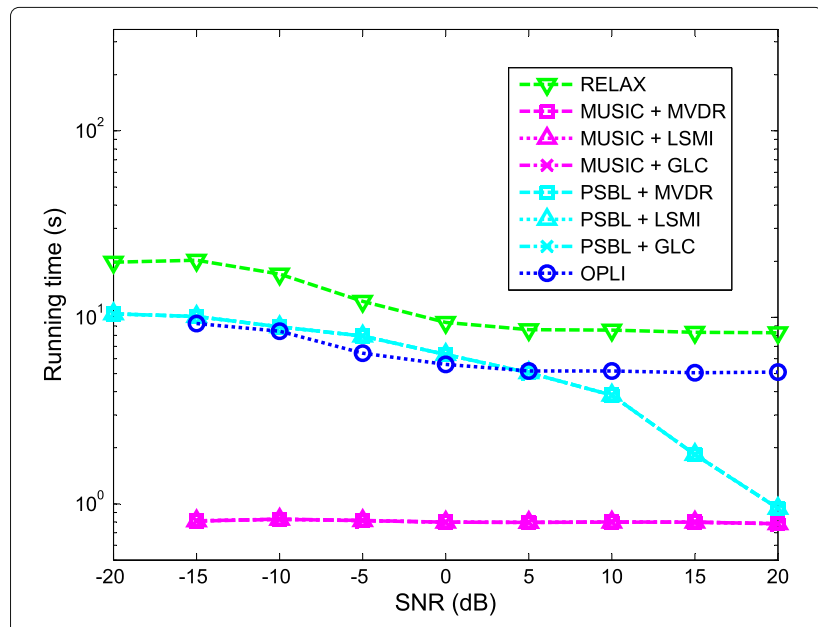

Fig. 12 Running time versus angular separation

Fig. 10 Running time at each input SNR 


\section{Methods}

The oblique projection-based localization and identification (OPLI) algorithm is proposed without known prior DOA or waveform information of the sources. The proposed OPLI employs oblique projection to separate the multiple incident signals into a series of single signal groups. Then, the source localization and waveform identification are implemented on each separated single signal. To this end, the OPLI is implemented iteratively. The method of maximum likelihood approximation is employed to evaluate the convergence.

\section{Conclusions}

A new OPLI algorithm, which is based on oblique projection, is proposed for localization and waveform identification of unknown target signal. The oblique projection is employed to separate the SOI from the received data of the array, and can also be used to suppress the interference signals at the same time.

The OPLI requires no prior information of the DOA or the signal waveforms, and it estimates the DOA and waveform of the SOI iteratively. Comparing to the well known RELAX and the combinational algorithms, which estimates DOA via employing MUSIC or PSBL, and realizes beamforming via employing MVDR, LSMI or GLC, the simulation results show that the proposed OPLI exhibits a better performance when the angular separation is small. Especially, when the input SNR within moderate to high region, the proposed OPLI not only shows an attractive output SINR which can coincide well with the optimal one, but also can achieve a high estimation accuracy for source localization since its RMSE could coincide well with CRB.

Both OPLI and RELAX are implemented iteratively, and the running time of the former is lower. Whereas, when compared to MUSIC-based counterpart combinational algorithms, the OPLI requires a higher computational complexity to implement source localization and waveform identification. Extensive experiments have been undertaken to verify the effectiveness and superiority of the OPLI with uncorrelated sources. Future work includes extension of the OPLI to correlated and coherent sources, and so on.

\section{Funding}

This work was supported by the National Natural Science Foundation of China (grant no. 61171180), by the Fundamental Research Funds for the Central Universities (grants nos. HIT. MKSTISP. 201613 and HIT. MKSTISP. 2016 26), and by a fund from the Science and Technology on Electronic Information Control Laboratory.

\section{Authors' contributions}

All authors read and approved the final manuscript.

\section{Competing interests}

The authors declare that they have no competing interests.

\section{Publisher's Note}

Springer Nature remains neutral with regard to jurisdictional claims in published maps and institutional affiliations.

\section{Author details}

${ }^{1}$ School of Electronics and Information Engineering, Harbin Institute of Technology, Harbin 150001, China. ${ }^{2}$ The No.14 Institute of CETC, Nanjing 210039, China. ${ }^{3}$ Qingdao Branch, Naval Aeronautical Engineering Institute, Qingdao 266041, China. ${ }^{4}$ Collaborative Innovation Centre of Information Sensing and Understanding, Harbin Institute of Technology, Harbin 150001, China. ${ }^{5}$ Science and Technology on Electronic Information Control Laboratory, Chengdu 610036, China.

Received: 16 September 2017 Accepted: 10 May 2018

Published online: 04 June 2018

\section{References}

1. S Sahnoun, P Comon, Joint source estimation and localization. IEEE Trans. Signal Process. 63(10), 2485-2495 (2015)

2. A Khabbazibasmenj, SA Vorobyov, A Hassanien, Robust adaptive beamforming based on steering vector estimation with as little as possible prior information. IEEE Trans. Signal Process. 60(6), 2974-2987 (2012)

3. H Krim, M Viberg, Two decades of array signal processing research: the parametric approach. IEEE Signal Process. Mag. 13(4), 67-94 (1996)

4. H Cox, R Zeskind, M Owen, Robust adaptive beamforming. IEEE Trans. Acoust. Speech Signal Process. ASSP-35(10), 1365-1375 (1987)

5. BD Van Veen, KM Buckley, Beamforming: a versatile approach to spatial filtering. IEEE ASSP Mag. 5(2), 4-24 (1988)

6. LC Godara, Application of antenna arrays to mobile communications, part II: beam-forming and direction-of-arrival considerations. Proc. IEEE. 85(8), 1195-1245 (1997)

7. RG Lorenz, SP Boyd, Robust minimum variance beamforming. IEEE Trans. Signal Process. 53(5), 1684-1696 (2005)

8. J Dmochowski, J Benesty, S Affes, Direction of arrival estimation using the parameterized spatial correlation matrix. IEEE/ACM Trans. Audio Speech Lang. Process. 15(4), 1327-1339 (2007)

9. JC Chen, K Yao, RE Hudson, Source localization and beamforming IEEE Signal Process. Mag. 19(2), 30-39 (2002)

10. Q Shen, W Liu, W Cui, SL Wu, Underdetermined DOA estimation under the compressive sensing framework: A review. IEEE Access. 4, 8865-8878 (2016)

11. X Liu, F Li, N Zhenyu, Optimal resource allocation in simultaneous cooperative spectrum sensing and energy harvesting for multichannel cognitive radio. IEEE Access. 5, 3801-3812 (2017)

12. $M$ Zhou, Y Tang, Z Tian, L Xie, W Nie, Robust neighborhood graphing for semi-supervised indoor localization with light-loaded location fingerprinting. IEEE Internet Things J. 99, 1-1 (2017)

13. M Zhou, Y Wei, Z Tian, X Yang, L Li, Achieving Cost-efficient Indoor Fingerprint Localization on WLAN Platform: A Hypothetical Test Approach. IEEE Access. 5, 15865-15874 (2017)

14. SA Vorobyov, AB Gershman, ZQ Luo, Robust adaptive beamforming using worst-case performance optimization: A solution to the signal mismatch problem. IEEE Trans. Signal Process. 51(2), 313-324 (2003)

15. B Liao, SC Chan, KM Tsui, Recursive steering vector estimation and adaptive beamforming under uncertainties. IEEE Trans. Aerosp. Electron. Syst. 49(1), 489-501 (2013)

16. JW Xu, GS Liao, SQ Zhu, L Huang, Response vector constrained robust LCMV beamforming based on semidefinite programming. IEEE Trans. Signal Process. 63(21), 5720-5732 (2015)

17. NY Wang, P Agathoklis, A Antoniou, A new DOA estimation technique based on subarray beamforming. IEEE Trans. Signal Process. 54(9), 3279-3290 (2006)

18. AZ Hu, DOA-based beamforming for multi-cell massive MIMO systems. J. Commun. Netw. 18(5), 735-743 (2016)

19. X Liu, XZ Tan, Optimization algorithm of periodical cooperative spectrum sensing in cognitive radio. Int. J. Commun. Syst. 27(5), 705-720 (2012)

20. A Haimovich, Y Bar-Ness, An eigenanalysis interference canceler. IEEE Trans. Signal Process. 39(1), 76-84 (1991)

21. A Haimovich, The eigencanceler: adaptive radar by eigenanalysis methods. IEEE Trans. Aerosp. Electron. Syst. 32(2), 532-542 (1996) 
22. ML Honig, JS Goldstein, Adaptive reduced-rank interference suppression based on the multistage wiener filter. IEEE Trans. Comput. 50(6), 986-994 (2002)

23. YJ Gu, A Leshem, Robust adaptive beamforming based on interference covariance matrix reconstruction and steering vector estimation. IEEE Trans. Signal Process. 60(7), 3881-3885 (2012)

24. L Huang, J Zhang, XXu, ZF Ye, Robust adaptive beamforming with a novel interference-plus-noise covariance matrix reconstruction method. IEEE Trans. Signal Process. 63(7), 1643-1650 (2015)

25. SC Chan, HH Chen, Uniform concentric circular arrays with frequencyinvariant characteristics - theory, design, adaptive beamforming and DOA estimation. IEEE Trans. Signal Process. 55(1), 165-177 (2007)

26. R Boyer, Oblique projection for source estimation in a competitive environment: algorithm and statistical analysis. Signal Process. 89(12), 2547-2554 (2009)

27. HB Xu, BR Zhu, J Liu, AZ Zhou, Robust beamforming design for secure multiuser MISO interference channel. IEEE Commun. Lett. 21(4), 833-836 (2017)

28. K Mak, A Manikas, A superresolution wide null beamformer for undersampled signal reconstruction in SIMO SAR. IEEE J. Sel. Top. Signal Process. 9(8), 1548-1559 (2015)

29. LM Shi, Y Lin, Convex combination of adaptive filters under the maximum correntropy criterion in impulsive interference. IEEE Signal Process. Lett. 21(11), 1385-1388 (2014)

30. RT Behrens, LL Scharf, Signal processing applications of oblique projection operators. IEEE Trans. Signal Process. 42(6), 1413-1424 (1994)

31. XP Mao, AJ Liu, J HH, Oblique projection polarisation filtering for interference suppression in high-frequency surface wave radar. IET Radar Sonar Navig. 6(2), 71-80 (2012)

32. XP Mao, YL Yang, H Hong, WB Deng, Multi-domain collaborative filter for interference suppressing. IET Signal Process. 10(9), 1157-1168 (2016)

33. A Hassanien, SA Elkader, AB Gershman, Convex optimization based beam-space preprocessing with improved robustness against out-of-sector sources. IEEE Trans. Signal Process. 54(5), 1587-1595 (2006)

34. RJ Vaccaro, BF Harrison, Optimal matrix-filter design. IEEE Trans. Signal Process. 44(3), 705-709 (1996)

35. D Han, XH Zhang, Optimal matrix filter design with application to filtering short data records. IEEE Signal Process. Lett. 17(5), 521-524 (2010)

36. M Souden, J Benesty, S Affes, A study of the LCMV and MVDR noise reduction filters. IEEE Trans. Signal Process. 58(9), 4925-4935 (2010)

37. Al Koutrouvelis, RC Hendriks, R Heusdens, J Jensen, Relaxed binaural LCMV beamforming. IEEE/ACM Trans. Audio Speech Lang. Process. 25(1), 137-152 (2017)

38. J Li, P Stoica, ZS Wang, On robust capon beamforming and diagonal loading. IEEE Trans. Signal Process. 51(7), 1702-1715 (2003)

39. L Du, J Li, P Stoica, Fully automatic computation of diagonal loading levels for robust adaptive beamforming. IEEE Trans. Aerosp. Electron. Syst. 46(1), 449-458 (2010)

40. SA Vorobyov, AB Gershman, ZQ Luo, Robust adaptive beamforming using worst-case performance optimization: a solution to the signal mismatch problem. IEEE Trans. Signal Process. 51(2), 313-324 (2003)

41. ZL Yu, W Ser, MH Er, et al, Robust adaptive beamformers based on worst-case optimization and constraints on magnitude response. IEEE Trans. Signal Process. 57(7), 2615-2628 (2009)

42. JR Guerci, Theory and application of covariance matrix tapers for robust adaptive beamforming. IEEE Trans. Signal Process. 47(4), 977-985 (1999)

43. $\mathrm{MHEr}, \mathrm{BC} \mathrm{Ng}, \mathrm{A}$ new approach to robust beamforming in the presence of steering vector errors. IEEE Trans. Signal Process. 42(7), 1826-1829 (1994)

44. F Shen, FF Chen, JY Song, Robust adaptive beamforming based on steering vector estimation and covariance matrix reconstruction. IEEE Commun. Lett. 19(9), 1636-1639 (2015)

45. H Li, YB Zhao, ZF Cheng, ZW Liu, PL Shui, Robust adaptive beamforming based on sparse representation technique. IET Radar Sonar Navig. 11(9), 1417-1424 (2017)

46. YX Ke, CS Zheng, RH Peng, XD Li, Robust adaptive beamforming using noise reduction preprocessing-based fully automatic diagonal loading and steering vector estimation. IEEE Access. 5, 12974-12987 (2017)

47. CJ Lam, AC Singer, Bayesian beamforming for DOA uncertainty: theory and implementation. IEEE Trans. Signal Process. 54(11), 4435-4445 (2006)

48. KL Bell, Y Ephraim, HL Van Trees, A Bayesian approach to robust adaptive beamforming. IEEE Trans. Signal Process. 48(2), 386-398 (2000)
49. YB Han, DQ Zhang, A recursive Bayesian beamforming for steering vector uncertainties. EURASIP J. Adv. Signal Process. 2013(108), 1-10 (2013)

50. J Li, P Stoica, Efficient mixed-spectrum estimation with applications to target feature extraction. IEEE Trans. Signal Process. 44(2), 281-295 (1996)

51. J Li, DM Zheng, P Stoica, Angle and waveform estimation via relax. IEEE Trans. Aerosp. Electron. Syst. 33(3), 1077-1087 (1997)

52. T Yardibi, J Li, P Stoica, M Xue, AB Baggeroer, Source localization and sensing: a nonparametric iterative adaptive approach based on weighted least squares. IEEE Trans. Aerosp. Electron. Syst. 46(1), 425-443 (2010)

53. I Ziskind, M Wax, Maximum likelihood localization of multiple sources by alternating projection. IEEE Trans. Acoust. Speech Signal Process. 36(10), 1553-1560 (1988)

54. P Stoica, A Nehorai, MUSIC, maximum likelihood, and Cramer-Rao bound. IEEE Trans. Acoust. Speech Signal Process. 37(5), 720-741 (1989)

55. RO Schmidt, Multiple emitter location and signal parameter estimation. IEEE Trans. Antennas Propag. 34(3), 276-280 (1986)

56. X Wu, WP Zhu, J Yan, Direction of arrival estimation for off-grid signals based on sparse bayesian learning. IEEE Sensors J. 16(7), 2004-2016 (2016)

\section{Submit your manuscript to a SpringerOpen ${ }^{\circ}$ journal and benefit from:}

- Convenient online submission

- Rigorous peer review

- Open access: articles freely available online

- High visibility within the field

- Retaining the copyright to your article

Submit your next manuscript at $>$ springeropen.com 